urgent, delayed, or expectant/deceased. The combination of these two methods will reduce the time spent with each casualty and make the entire triage process much more rapid.

Background: With an increase in mass shootings in recent years, providers may find themselves responding to an active shooter scenario incident. Lessons learned from the incidents in the Paris theater shooting, Columbine school shooting, and Orlando nightclub shooting demonstrate the lapses in active shooter and triage protocols.

Methods: In those with an inability to follow basic commands, in addition to a weak or absent radial pulse, the mortality rate approaches $92 \%$. In a mass shooting event, a novel "two step" triage technique is proposed, in which EMS determines if the casualty can follow a basic command, such as "squeeze my fingers," combined with the assessment of a radial pulse to establish a triage category.

Results: The benefit of this simplified process is speed and ease of use. When there are dozens of patients, spending 60 seconds with each patient is not only unfeasible; it may cost the lives of those awaiting triage.

Conclusion: The current method of responding to active shooter incidents is ineffective at best, and current triage methods are overly complicated and difficult to implement. By moving toward new triage and scene management techniques, the potential to save numerous lives is possible.

Prehosp Disaster Med 2017;32(Suppl. 1):s12-s13

doi:10.1017/S1049023X17000589

Evacuating a Geriatric Medical Facility during Emergencies - Exercising a Complex Challenge

R. Ringel ${ }^{1}$, A. Ohana ${ }^{1}$, E. Furman ${ }^{2}$, B. Adini ${ }^{3}$

1. Ministry of Health, Jerusalem/Israel

2. Medical Department, Israel Defense Forces Home Front Command, Kfar Monash/Israel

3. Disaster Management \& Injury Prevention, Tel Aviv University, Tel Aviv/Israel

Study/Objective: To examine lessons learned from exercises simulating evacuation of geriatric hospitals to improve emergency preparedness.

Background: Emergency events may necessitate full or partial evacuation of geriatric hospitals, posing a complex challenge. The evacuation process entails close medical supervision of chronic/ventilated patients and provision of oxygen-support means in tandem with evacuation of visitors and hospital staff. In order to generate an effective automatic response to such situations, the Ministry of Health (MOH) developed a national evacuation doctrine of geriatric facilities and evaluates it through routine exercises.

Methods: The geriatric evacuation exercises encompass four main processes: decision-making, planning, implementing the evacuation, and return to normalcy. Following each exercise, an After Action Review is conducted, to identify elements that should be sustained or improved.

Results: Strategic, operational and infrastructural lessons were learnt, including: 1) evacuate patients based on internally available personnel and equipment rather than reinforcements; 2) accompany each evacuating vehicle with hospital's team member to ensure patients' safe arrival to a receiving facility including sharing information; 3) plan and utilize designated forms, to facilitate control and monitoring of the evacuation; 4) equip each evacuation vehicle with a list of the patients including contact details of evacuating and receiving facilities; 5) operate an "evacuation operation center;" 6) prepare a mechanism to assess patients that may potentially be released home; 7) plan evacuation of patients through stairs, due to potential dysfunctional elevators; 8) plan positioning of rescue forces in a way that will prevent blockage of evacuation routes; 9) exercise joint communication means to ensure flow of information between all responders.

Conclusion: Evacuation exercises significantly promote emergency preparedness of geriatric hospitals and strengthen their collaboration with first responders. Readiness of geriatric hospitals for an emergency evacuation necessitates preparedness of resources, life-saving equipment, and personnel to facilitate a rapid response during such a complex emergency.

Prehosp Disaster Med 2017;32(Suppl. 1):s13

doi:10.1017/S1049023X17000590

\section{Conflict and Disaster Medicine: The State of Military} Medicine in Ukraine

John M. Quinn $V^{1}$, Vladimir Bencko ${ }^{2}$, Patrick Chellew ${ }^{3}$, Olia Romaniuk ${ }^{4}$

1. Expert Consultant, Reforms Office (ro) At The Ministry Of Defense (mod) In Ukraine, PhD Candidate and Researcher, Prague Center for Global Health, Institute of Hygiene and Epidemiology, First Faculty of Medicine, Charles University, Praha/Czech Republic

2. Prague Center for Global Health, Institute of Hygiene and Epidemiology, First Faculty of Medicine, Charles University in Prague, Praha/Czech Republic

3. Program Lead, Medical ATO Report Series, Kyiv/Ukraine

4. Program Researcher, Medical ATO Report Series, Kyiv/Ukraine

Study/Objective: Assess battlefield morbidity and mortality, from point of injury to tertiary care, across the spectrum of the evacuation chain. This is to include phases of TCCC and Prolonged Field Care (PFC) for war-fighting and ancillary activities in Eastern Ukraine and peri-conflict regions.

Background: The former Ukrainian president Yanakovich's refusal to sign an agreement, bringing Ukraine economically closer to the EU in November 2013, ignited a political and social revolution. The reforms' process for the Ministry of Defense $(\mathrm{MoD})$ is a long struggle, and as NATO alignment in all processes remains the goal, multiple actors must continue support it in order to save life, enable the soldier to have access to best practices in battlefield medicine, maintain state sovereignty, promote democracy, and uphold the Ukrainian constitutional values.

Methods: Qualitative and quantitative morbidity and mortality data from war-fighting activity; research spanning 2014-2016. Field data in the form of structured interviews and surveys, core methods under the Working Group model, in collaboration with all departments and institutes at the military medical academy.

Results: We conclude that the evacuation chain has improved markedly from the onset of the war, but that hybrid warfare by 